\title{
A High-Performance CMOS Voltage-Controlled Oscillator for Ultra-Low-Voltage Operations
}

\author{
Hsieh-Hung Hsieh, Student Member, IEEE, and Liang-Hung Lu, Member, IEEE
}

\begin{abstract}
In this paper, a novel circuit topology of voltage-controlled oscillators (VCOs) suitable for ultra-low-voltage operations is presented. By utilizing the capacitive feedback and the forward-body-bias (FBB) technique, the proposed VCO can operate at reduced supply voltage and power consumption while maintaining remarkable circuit performance in terms of phase noise, tuning range, and output swing. Using a standard 0.18- $\mu \mathrm{m}$ CMOS process, a 5.6-GHz VCO is designed and fabricated for demonstration. Consuming a de power of $3 \mathrm{~mW}$ from a 0.6-V supply voltage, the VCO exhibits a frequency tuning range of $8.1 \%$ and a phase noise of $-118 \mathrm{dBc} / \mathrm{Hz}$ at $1-\mathrm{MHz}$ offset frequency. With an FBB for the cross-coupled transistors, the fabricated circuit can operate at a supply voltage as low as $0.4 \mathrm{~V}$. The measured tuning range and phase noise are $6.4 \%$ and $-114 \mathrm{dBc} / \mathrm{Hz}$, respectively.
\end{abstract}

Index Terms-Capacitive feedback, forward body bias (FBB), ultra-low power, ultra-low voltage, voltage-controlled oscillators (VCOs).

\section{INTRODUCTION}

$\mathbf{W}$ ITH THE emerging applications such as wireless personal area networks (WPANs), wireless sensor networks and RF identifications (RFIDs), the development of low-cost and low-power RF integrated circuits (RFICs) for short-range communications have attracted great attention over the past few years. For the circuit implementations, the CMOS technology appears to be particularly well suited due to its unparalleled advantages in the fabrication cost and system-level integration. Unfortunately, the inherently low transconductance of the MOSFETs at higher frequencies have impeded the evolution of low-power designs to RF front-ends. In order to overcome the limitations, various design methodologies and circuit techniques have been proposed [1]-[4]. Among these approaches, low-voltage circuit operation is one of the most promising solutions. In addition to the power considerations, a reduced supply voltage is also an inevitable trend for CMOS designs as well. With the continuous shrinking in the transistor feature size, a proportional down-scaling of the supply is required to ensure the gate-oxide reliability [5]. The forecast of the supply voltage for CMOS circuits within the next decade [6] is shown in Fig. 1, where a low-voltage operation of $0.4 \mathrm{~V}$ is anticipated by the end of 2020. Therefore, there exists an urgent need to develop low-voltage circuit techniques for high-performance RFICs at multigigahertz frequencies.

Manuscript received July 10, 2006; revised November 20, 2006. This work was supported in part by the National Science Council under Grant 94-2220-E002-026 and Grant 94-2220-E-002-009.

The authors are with the Graduate Institute of Electronics Engineering and Department of Electrical Engineering, National Taiwan University, Taipei 10617, Taiwan, R.O.C. (e-mail: 1hlu@cc.ee.ntu.edu.tw).

Digital Object Identifier 10.1109/TMTT.2006.89147

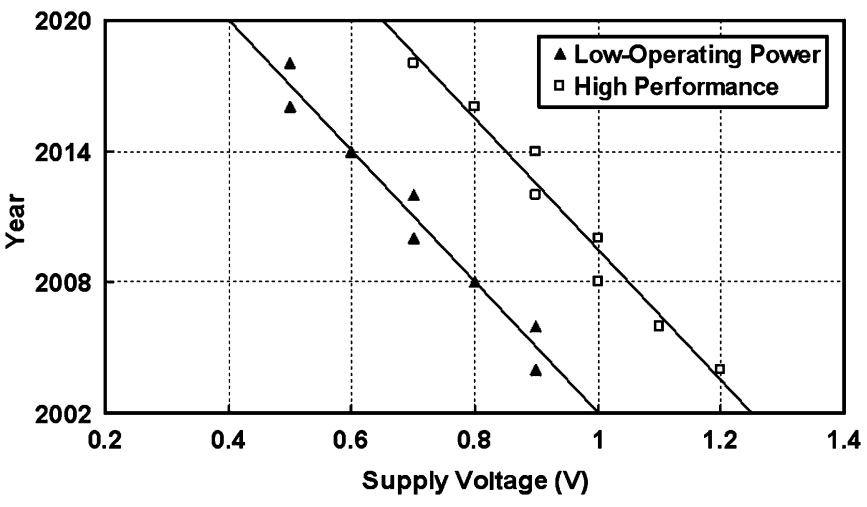

Fig. 1. Forecast of the CMOS supply voltage by International Technology Roadmap for Semiconductors (ITRS) 2004.

Being a crucial part in the RF front-ends, the voltage-controlled oscillator (VCO) is considered as one of the most power-consuming components. For low-power applications, CMOS oscillators operating at a supply voltage lower than $1 \mathrm{~V}$ were reported [7]-[11]. However, most of the circuits suffer from reduced output swing and degraded phase noise due to the limitations on the supply voltage. In this study, a VCO topology suitable for ultra-low-power and ultra-low-voltage operations is presented. By incorporating the capacitive feedback and the forward-body-bias (FBB) technique in the cross-coupled CMOS VCO, remarkable circuit performance in terms of the phase noise, frequency tuning range, and output swing can be achieved. Using a $0.18-\mu \mathrm{m}$ CMOS technology, a $5.6-\mathrm{GHz}$ VCO is implemented for demonstration.

The remainder of this paper is organized as follows. Section II describes the proposed circuit topology and the design considerations. The circuit design and the experimental results of the 5.6-GHz CMOS VCO are presented in Sections III and IV, respectively. Finally, a conclusion is given in Section V.

\section{PROPOSED VCO TOPOLOGY}

The schematic of the proposed VCO is shown in Fig. 2. In order to reduce the required supply voltage and to eliminate additional noise contribution, the tail current transistor in a conventional cross-coupled VCO is replaced by on-chip inductors [12]. For an enhanced voltage swing under an ultra-low supply voltage, the capacitive-feedback technique is employed [13]. Due to the use of the on-chip inductor and the feedback loop established by $C_{1}$ and $C_{2}$, the drain and source voltages can swing above the supply voltage and below the ground potential. Consequently, the output swing of the VCO is enhanced, leading 


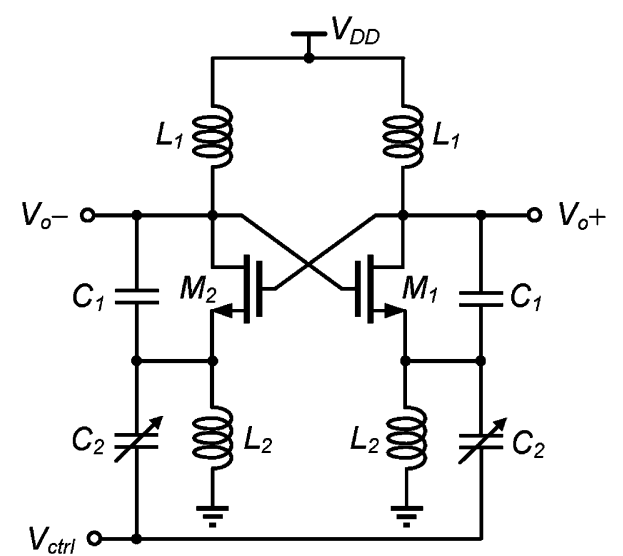

Fig. 2. Schematic of the proposed VCO topology.

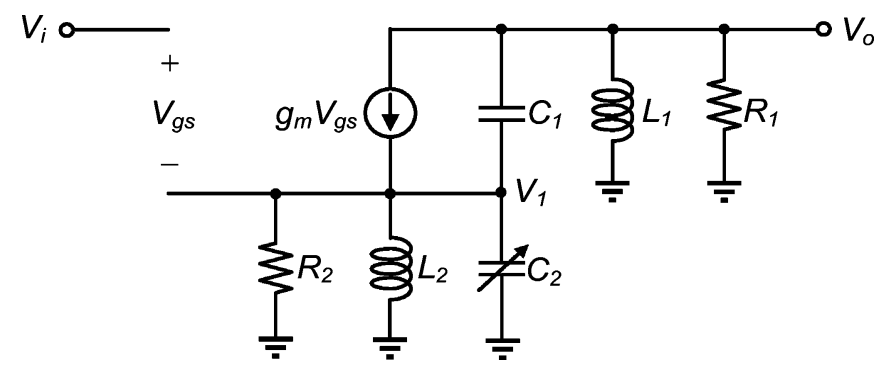

Fig. 3. Simplified half-circuit model of the proposed VCO.

to a superior close-in phase noise. Since the varactors are employed in the source terminals of the cross-coupled transistors, a more effective controlled mechanism of the tank resonance is presented. Therefore, a reasonable frequency tuning range can be achieved even with a reduced voltage range for the controlled signal $V_{\text {ctrl }}$. To further investigate the proposed VCO, detailed circuit analysis is provided as follows.

\section{A. Startup Conditions}

In order to derive the startup conditions and the oscillation frequency, the equivalent half-circuit of the proposed VCO is shown in Fig. 3, where $R_{1}$ and $R_{2}$ represent the losses of the on-chip inductors $L_{1}$ and $L_{2}$, respectively. Note that the losses of the inductors are typically modeled by a series resistance. In the equivalent circuit, the narrowband approximation is employed to simplify the analysis, and the shunt resistance $R_{1}$ and $R_{2}$ can be estimated by

$$
\begin{aligned}
& R_{1} \approx \omega^{2} L_{1}^{2} / R_{s 1} \\
& R_{2} \approx \omega^{2} L_{2}^{2} / R_{s 2}
\end{aligned}
$$

where $R_{s 1}$ and $R_{s 2}$ are the equivalent series resistances of $L_{1}$ and $L_{2}$, respectively. Besides, the transistor parasitic capacitances, which are much smaller than the values of $C_{1}$ and $C_{2}$, are neglected. From the small-signal analysis, the transfer function between $V_{o}$ and $V_{i}$ is given by

$$
\frac{V_{o}}{V_{i}}=\frac{-g_{m}\left(L_{1} L_{2} R_{1} R_{2} C_{2} s^{2}+L_{1} L_{2} R_{1} s+L_{1} R_{1} R_{2}\right) s}{a_{4} s^{4}+a_{3} s^{3}+a_{2} s^{2}+a_{1} s+a_{0}}
$$

where

$$
\begin{aligned}
a_{0} & =R_{1} R_{2} \\
a_{1} & =L_{1} R_{2}+L_{2} R_{1}+g_{m} L_{2} R_{1} R_{2} \\
a_{2} & =L_{1} L_{2}\left(1+g_{m} R_{2}\right)+R_{1} R_{2}\left(L_{1} C_{1}+L_{2} C_{1}+L_{2} C_{2}\right) \\
& \approx R_{1} R_{2}\left(L_{1} C_{1}+L_{2} C_{1}+L_{2} C_{2}\right) \\
a_{3} & =L_{1} L_{2}\left(R_{1} C_{1}+R_{2} C_{1}+R_{2} C_{2}\right) \\
a_{4} & =L_{1} L_{2} R_{1} R_{2} C_{1} C_{2} .
\end{aligned}
$$

The circuit oscillates if the loop gain is unity, which corresponds to a voltage gain $V_{o} / V_{i}=-1$ at the oscillation frequency $\omega_{0}$

$$
\frac{-j g_{m} \omega_{0}\left(-L_{1} L_{2} R_{1} R_{2} C_{2} \omega_{0}^{2}+j L_{1} L_{2} R_{1} \omega_{0}+L_{1} R_{1} R_{2}\right)}{a_{4} \omega_{0}^{4}-j a_{3} \omega_{0}^{3}-a_{2} \omega_{0}^{2}+j a_{1} \omega_{0}+a_{0}}=-1 .
$$

With proper arrangement, (4) yields

$$
L_{1} L_{2} C_{1} C_{2} \omega_{0}^{4}-\left[L_{1} C_{1}+L_{2}\left(C_{1}+C_{2}\right)-\frac{L_{1} L_{2} g_{m}}{R_{2}}\right] \omega_{0}^{2}+1=0
$$

and

$$
\begin{array}{r}
{\left[L_{1} L_{2}\left(R_{1} C_{1}+R_{2} C_{1}+R_{2} C_{2}\right)-g_{m} L_{1} L_{2} R_{1} R_{2} C_{2}\right] \omega_{0}^{3}} \\
-\left[g_{m} R_{1} R_{2}\left(L_{2}-L_{1}\right)+L_{1} R_{2}+L_{2} R_{1}\right] \omega_{0}=0 .
\end{array}
$$

Provided $g_{m} L_{1} \ll R_{2}\left(C_{1}+C_{2}\right)$ in typical design cases, (5) can be simplified as

$$
L_{1} L_{2} C_{1} C_{2} \omega_{0}^{4}-\left[L_{1} C_{1}+L_{2}\left(C_{1}+C_{2}\right)\right] \omega_{0}^{2}+1=0 .
$$

From (7), the oscillation frequency can be approximated by

$$
\omega_{0} \approx \sqrt{\frac{1}{L_{2} C_{2}}+\frac{C_{1}+C_{2}}{L_{1} C_{1} C_{2}}} .
$$

Based on (6) and (8), the required transconductance $g_{m}$ to sustain the oscillation is given by

$$
g_{m}=\frac{L_{1} L_{2}\left(R_{1} C_{1}+R_{2} C_{1}+R_{2} C_{2}\right) \omega_{0}^{2}-L_{1} R_{2}-L_{2} R_{1}}{R_{1} R_{2}\left(L_{2}-L_{1}\right)+L_{1} L_{2} R_{1} R_{2} C_{2} \omega_{0}^{2}} .
$$

Assuming that $L_{1}=L_{2}=L_{p}$ and $R_{1}=R_{2}=R_{p}$, (8) and (9) can be simplified as

$$
\begin{aligned}
\omega_{0} & =\sqrt{\frac{1}{L_{p}}\left(\frac{1}{C_{1}}+\frac{2}{C_{2}}\right)} \\
g_{m} R_{p} & =1+\frac{4\left(C_{1} / C_{2}\right)^{2}}{1+2\left(C_{1} / C_{2}\right)} .
\end{aligned}
$$

From (10), it is clear that the variation of $C_{2}$ has more influence on $\omega_{0}$ than $C_{1}$ does. As a result, the capacitance $C_{2}$ is realized by varactors in this design, leading to a reasonable tuning range for the VCO under ultra-low-voltage operations. Note that a large capacitance value for $C_{1}$ is desirable to enhance the frequency tuning range. However, the required transconductance to sustain the oscillation increases with the ratio of $C_{1}$ and $C_{2}$. Therefore, a design tradeoff is established between the frequency tuning range and the power consumption of the VCO by the circuit parameter $C_{1} / C_{2}$. Once the capacitance ratio is determined, the required transconductance of the cross-coupled 


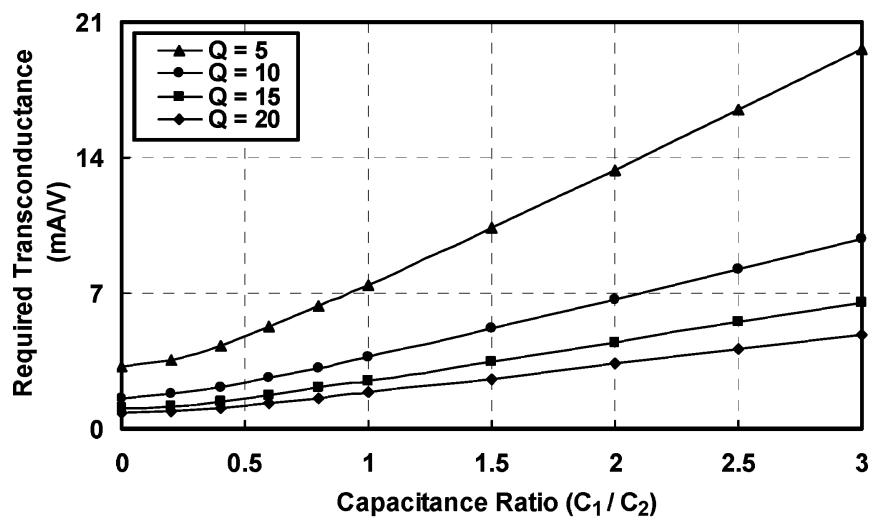

Fig. 4. Required transconductance to satisfy the startup conditions for various inductor $Q$ factors.

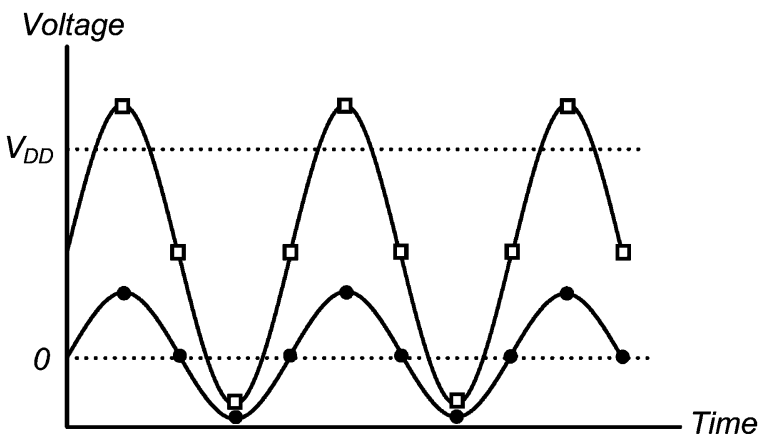

\section{—— Drain Voltage Swing}

$\longrightarrow \quad$ Source Voltage Swing

Fig. 5. Conceptual illustration of the drain and source voltage due to the capacitive feedback.

transistors can be estimated by (11). Assuming an inductance value of $2 \mathrm{nH}$ and an oscillation frequency of $5 \mathrm{GHz}$, the required transconductance as a function of $C_{1} / C_{2}$ for various inductor quality factors are shown in Fig. 4, which provides useful design guidelines for the proposed circuit topology.

\section{B. Output Voltage Swing}

In the proposed VCO circuit, a capacitive feedback is formed by capacitors $C_{1}$ and $C_{2}$. Due to the in-phase relationship provided by the capacitive feedback and the use of on-chip inductors, the drain and source voltage can swing above the supply voltage and below the ground potential, as illustrated in Fig. 5 . Consequently, the close-in phase noise benefits from the enhanced voltage swing at the VCO output. To evaluate the performance enhancement of this technique, the output voltage swing of the VCO is derived from the time-domain waveform of the drain current $I_{1}(t)$, as shown in Fig. 6. For simplicity, the periodic drain current is modeled by a square wave with a period of $T_{0}=2 \pi / \omega_{0}$ and an amplitude of $I_{0}$. Note that, at the quiescent point, the transistors are biased at $V_{D 1}=V_{D 2}=V_{D D}$ and $V_{S 1}=V_{S 2}=0$. The maximum drain current $I_{1}(t)$ occurs when the gate voltage $V_{G 1}$ reaches its peak value. Assuming that the amplitude of output oscillating signal is $A$, the gate voltages $V_{G 1}$ and $V_{G 2}$ are $\left(V_{D D}+A\right)$ and $\left(V_{D D}-A\right)$, respectively, while the

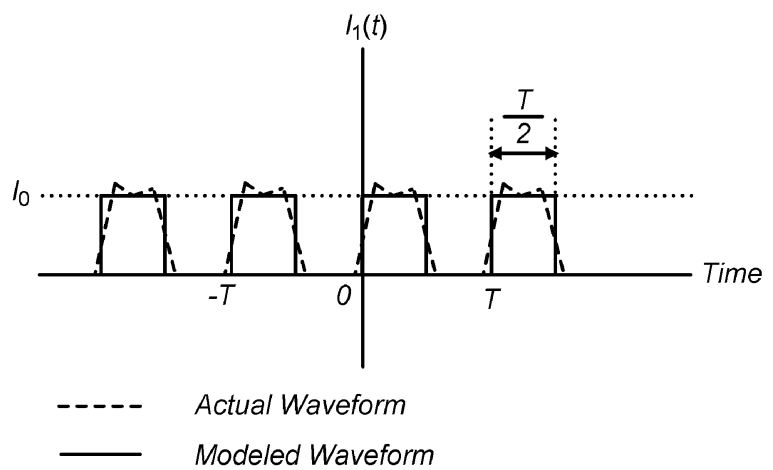

Fig. 6. Actual and modeled drain current waveform of the transistor $M_{1}$.

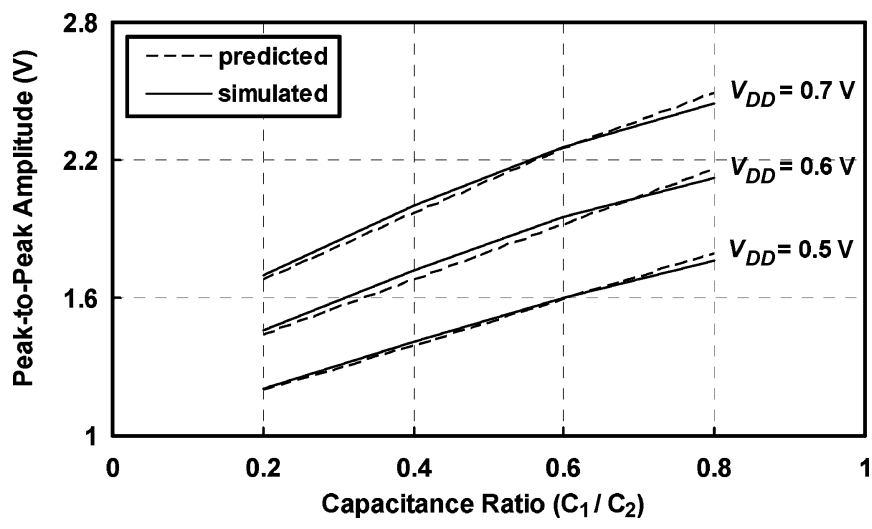

Fig. 7. Predicted and simulated peak-to-peak amplitude at the VCO output.

source voltages $V_{S 1}$ and $V_{S 2}$ can be obtained by the voltage divider of $C_{1}$ and $C_{2}$. Thus, $I_{0}$ is approximated by the maximum drain current with the transistor operating in the nonsaturated region

$$
\begin{array}{r}
I_{0} \approx \mu_{n} C_{\mathrm{ox}} \frac{W}{L}\left[\left(V_{D D}+A+\right.\right. \\
\left.n A-V_{t}\right)\left(V_{D D}-A+n A\right) \\
\left.-\frac{1}{2}\left(V_{D D}-A+n A\right)^{2}\right]
\end{array}
$$

where $n=C_{1} /\left(C_{1}+C_{2}\right)$ and $V_{t}$ is the threshold voltage of the MOSFET. From the Fourier series of $I_{1}(t)$, the fundamental current component is given by

$$
\left.I_{1}(t)\right|_{\text {fundamental }} \approx \frac{2}{\pi} I_{0} \sin \left(\omega_{0} t\right)
$$

and the fundamental voltage amplitude is

$$
A \approx \frac{2}{\pi} I_{0} R_{p}
$$

where $R_{p}$ is the load resistance. From (12) and (14), a simplified expression of the VCO output swing is given by

$$
A \approx\left(1+C_{1} / C_{2}\right) \cdot V_{D D} .
$$

According to (15), the predicted VCO output amplitude as a function of $C_{1} / C_{2}$ is depicted in Fig. 7 along with the values obtained from the circuit-level simulation. It is obvious that an output swing significantly larger than the supply voltage can be achieved by the proposed capacitive feedback. 


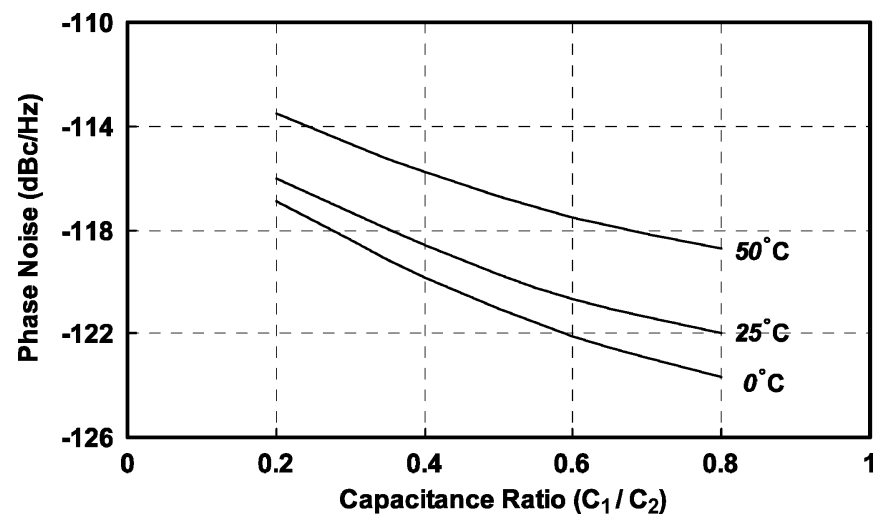

Fig. 8. Simulated phase noise at various temperatures with an offset frequency of $1 \mathrm{MHz}$.
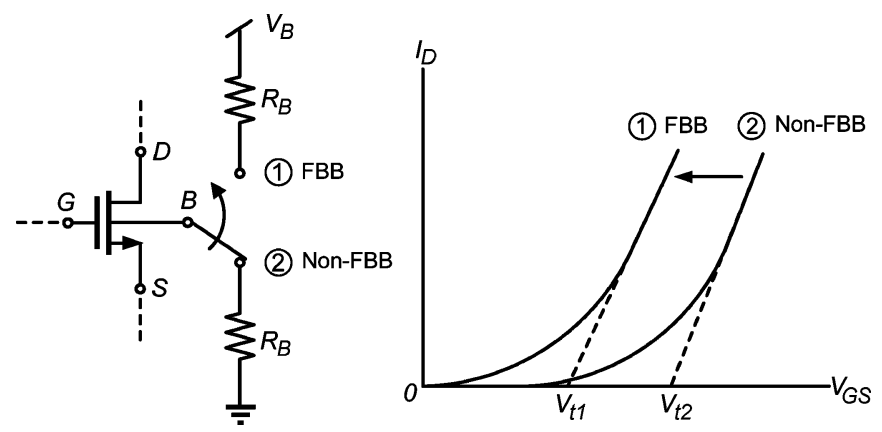

Fig. 9. $I-V$ characteristics of the MOSFET with and without FBB.

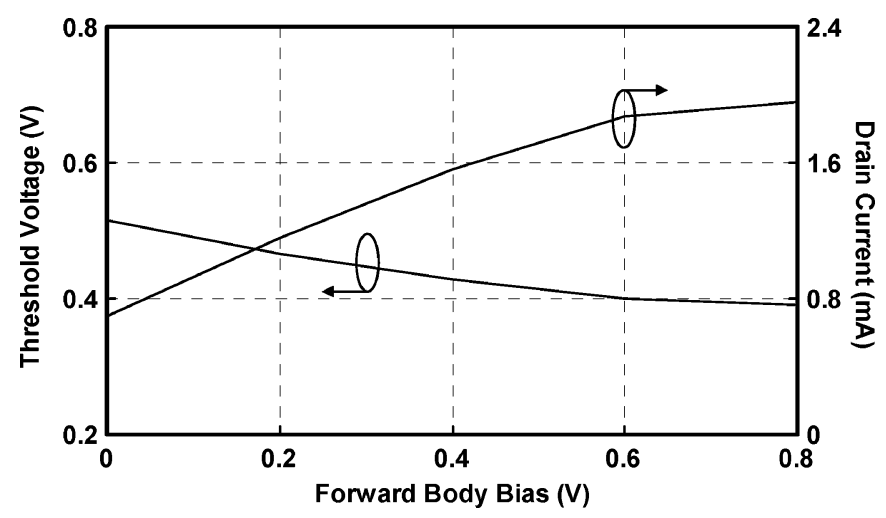

Fig. 10. Simulated threshold voltage and drain current of the MOSFET with FBB.

\section{Phase Noise}

Since the proposed VCO is operated under an ultra-low dc voltage, the cross-coupled transistors are potentially biased in the weak-inversion region. Therefore, the drain noise of the transistors is no longer dominated by the thermal noise as [14]

$$
\frac{i_{n, d}}{\Delta f}=4 k T \gamma g_{d 0}
$$

where $g_{d 0}$ is the channel conductance with $V_{\mathrm{GS}}=0$, and $\gamma$ is the thermal noise coefficient. Instead, the drain noise is expressed

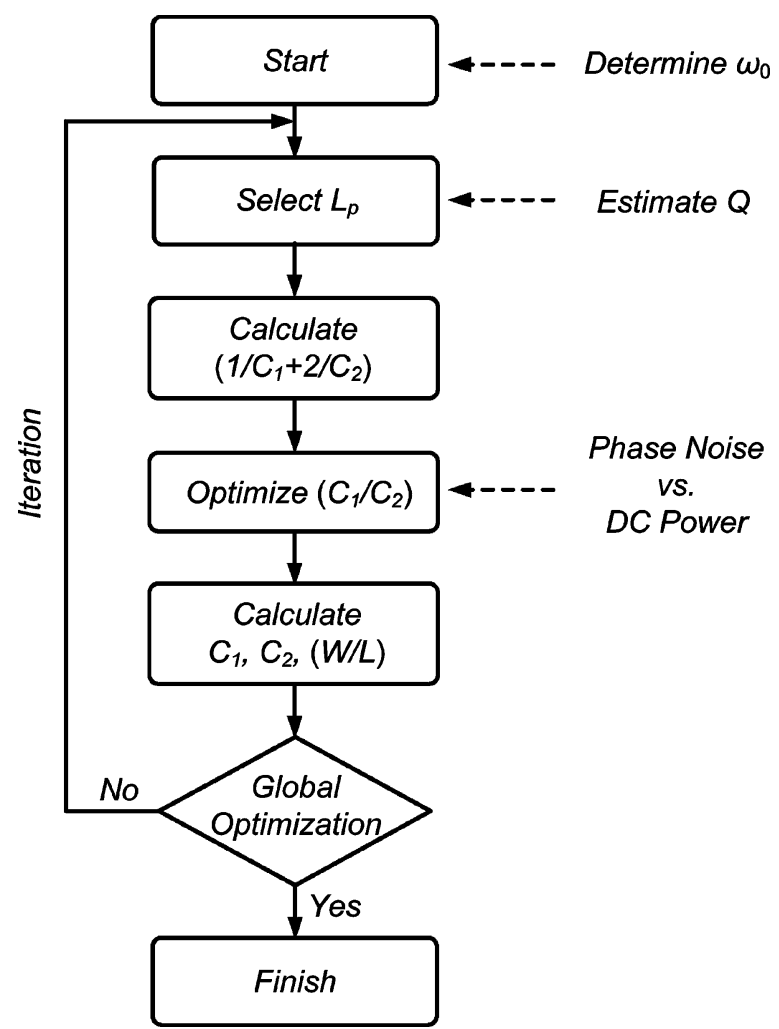

Fig. 11. Proposed methodology for the design of the low-voltage VCO.

TABLE I

CirCUIT PARAMETERS OF THE VCO

\begin{tabular}{|c|c|c|}
\hline Devices & \multicolumn{2}{|c|}{ Design Values } \\
\hline$M_{1}, M_{2}$ & \multicolumn{2}{|c|}{$35 \mu \mathrm{m} / 0.18 \mu \mathrm{m}$} \\
\hline \multirow{2}{*}{$L_{1}, L_{2}$} & $L_{p}$ & $1.5 \mathrm{nH}$ \\
\cline { 2 - 3 } & $Q$ & 12 \\
\hline$C_{1}$ & \multicolumn{2}{|c|}{$0.68 \mathrm{pF}$} \\
\hline$C_{2}$ & \multicolumn{2}{|c|}{$1 \sim 2 \mathrm{pF}$} \\
\hline$C_{1} / C_{2}$ & \multicolumn{2}{|c|}{$0.34 \sim 0.68$} \\
\hline$g_{m}$ & \multicolumn{2}{|c|}{$8 \mathrm{~mA} / \mathrm{V}$} \\
\hline
\end{tabular}

as [15]

$$
\frac{i_{n, d}}{\Delta f}=\frac{4 k T \gamma g_{d o}}{1+\exp \left(-\frac{V_{\mathrm{GS}}-V_{t}}{2 m V_{T}}\right)}+\frac{2 q I_{D}}{1+\exp \left(\frac{V_{\mathrm{GS}}-V_{t}}{2 m V_{T}}\right)}
$$

where $I_{D}$ is the drain current, $V_{T}$ is the thermal voltage, and $m$ is the weak inversion slope factor. From (17), $i_{n, d} / \Delta f$ includes not only the contribution from the thermal noise, but also that of the shot noise since the drain current consists of both drift and diffusion components as the gate overdrive $V_{\mathrm{GS}}-V_{t}$ approaches $V_{T}$. Due to the fact that $2 q I_{D}$ is generally greater than $4 k T \gamma g_{d 0}$ for low-power operation, the cross-coupled transistors may contribute more noise to the $L C$ tank as the supply voltage decreases. Therefore, it imposes a fundamental limitation on the phase noise of the VCO for ultra-low-power and ultra-low-voltage applications. 


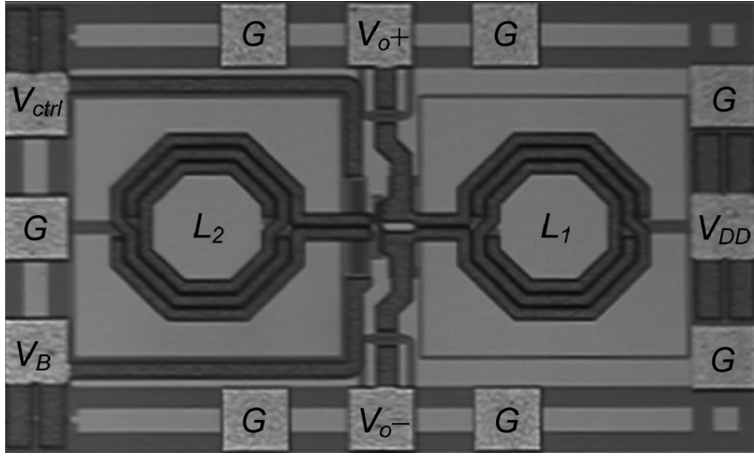

Fig. 12. Microphotograph of the fabricated VCO.

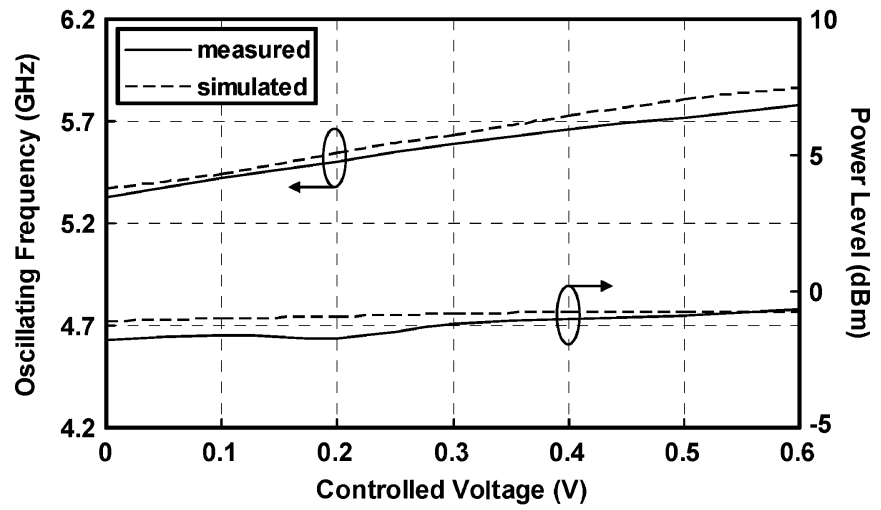

Fig. 13. Measured and simulated tuning characteristics of the VCO with a supply voltage of $0.6 \mathrm{~V}$.

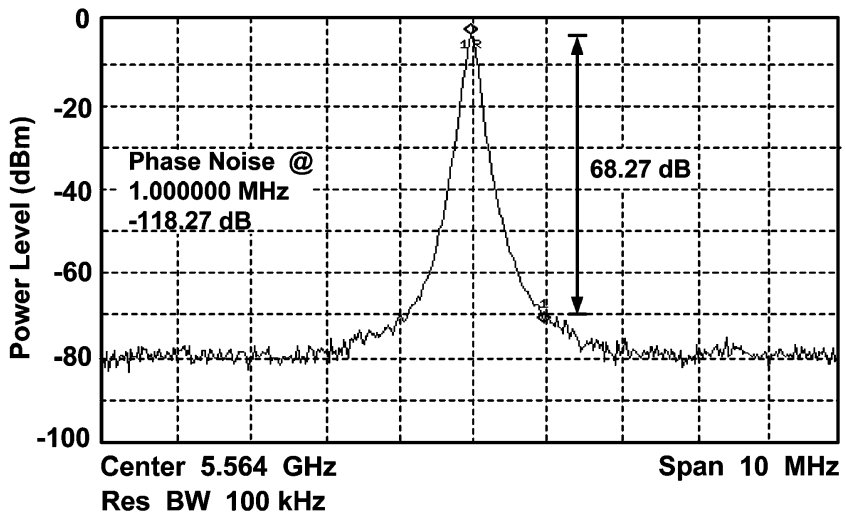

Fig. 14. Measured close-in output spectrum of the VCO with a supply voltage of $0.6 \mathrm{~V}$.

For the conventional $L C$-tank VCOs operating at a reduced supply voltage, a significant degradation in the phase noise is inevitable due to the limited signal power at the output. In the proposed VCO topology, the output swing can be effectively enhanced by the capacitive feedback, leading to an improved close-in phase noise. Furthermore, due to the similarity to the Colpitts oscillator, the VCO circuit also benefits from the cyclostationary noise effect [16]. To further investigate the influence of the capacitive feedback, the simulated phase noise as a function of the capacitance ratio $C_{1} / C_{2}$ is shown in Fig. 8. It is noted that lower phase noise can be achieved by increasing the capacitance ratio. However, as shown in (11), the performance improvement in the phase noise is accompanied by an increase

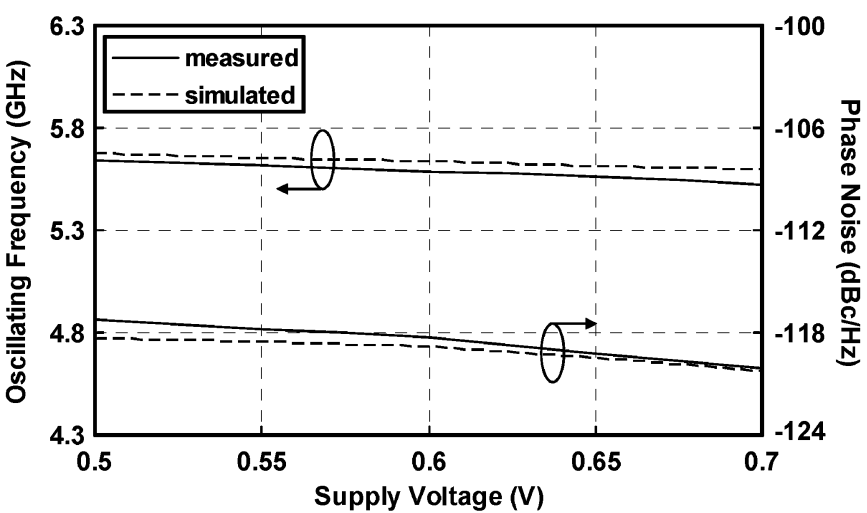

Fig. 15. Measured and simulated VCO characteristics with a supply voltage sweeping from 0.5 to $0.7 \mathrm{~V}$.

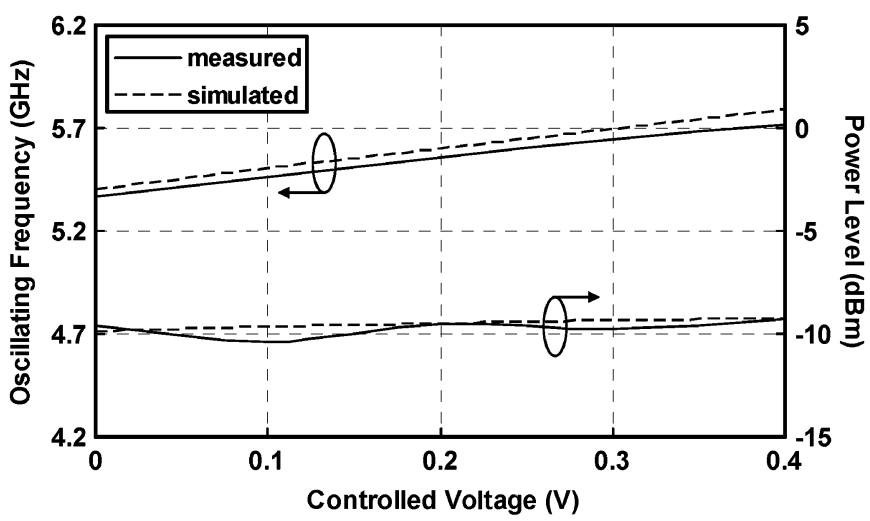

Fig. 16. Measured and simulated tuning characteristics of the VCO with a supply voltage and body bias of $0.4 \mathrm{~V}$.

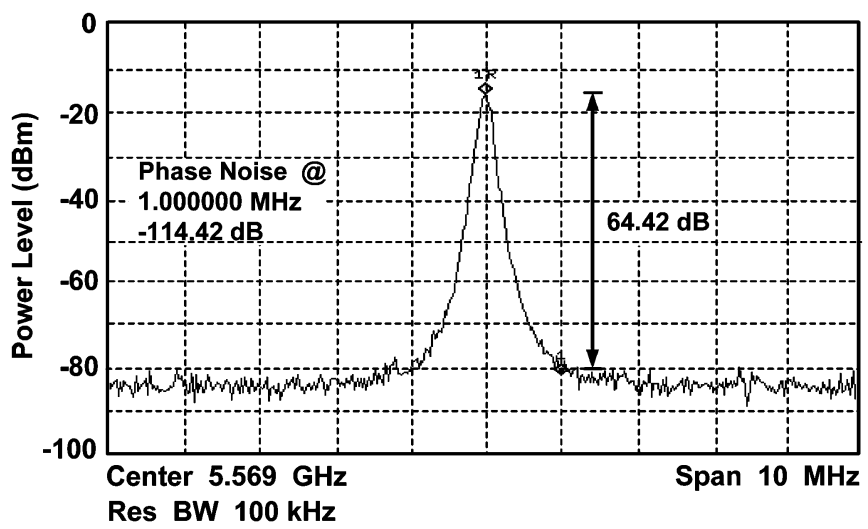

Fig. 17. Measured close-in output spectrum of the VCO with a supply voltage and body bias of $0.4 \mathrm{~V}$.

in the required transconductance, which implies higher power consumption of the VCO circuit. In addition to the capacitance ratio, the temperature also influences the VCO phase noise. The simulated results at various temperatures are illustrated in Fig. 8 as well, indicating an average $4-\mathrm{dB}$ degradation in phase noise as the temperature increases from $25^{\circ} \mathrm{C}$ to $50{ }^{\circ} \mathrm{C}$.

\section{D. $F B B$}

For deep-submicrometer MOSFETs, the threshold voltage $V_{t}$ is no longer constant, but influenced by circuit parameters such as gate length, channel width, and drain-to-source voltage due 
TABLE II

PERFORMANCE SUMmaRY OF THE LOW-VOLTAGE AND LOW-POWER VCOS

\begin{tabular}{|c|c|c|c|c|c|c|c|c|}
\hline & Unit & \multicolumn{2}{|c|}{ This Work } & [7] & [19] & [20] & \multicolumn{2}{|c|}{ [8] } \\
\hline Technology & - & \multicolumn{2}{|c|}{$\begin{array}{l}0.18-\mu \mathrm{m} \\
\text { CMOS }\end{array}$} & $\begin{array}{c}0.18-\mu \mathrm{m} \\
\mathrm{CMOS}\end{array}$ & $\begin{array}{l}0.18-\mu \mathrm{m} \\
\text { CMOS }\end{array}$ & $\begin{array}{c}0.18-\mu \mathrm{m} \\
\text { CMOS }\end{array}$ & \multicolumn{2}{|c|}{$\begin{array}{c}0.18-\mu \mathrm{m} \\
\text { CMOS }\end{array}$} \\
\hline Frequency & $\mathrm{GHz}$ & \multicolumn{2}{|c|}{5.6} & 5.8 & 4.5 & 4.8 & 1.4 & 3.8 \\
\hline Tuning Range & $\%$ & 8.1 & 6.4 & 8.9 & 4.4 & $4.3^{3}$ & 0 & 3.0 \\
\hline $\begin{array}{c}\text { Phase Noise } \\
\text { @1-MHz }\end{array}$ & $\mathrm{dBc} / \mathrm{Hz}$ & -118 & -114 & -97 & $-100^{2}$ & -120 & -129 & -119 \\
\hline $\mathrm{V}_{\mathrm{DD}}$ & $\mathrm{V}$ & 0.6 & 0.4 & 0.6 & 0.8 & 1.5 & 0.35 & 0.5 \\
\hline DC power & $\mathrm{mW}$ & 3.0 & 1.1 & 0.7 & - & 3.0 & 1.5 & 0.6 \\
\hline Output Power Level & $\mathrm{dBm}$ & -1 & -9 & -10 & - & 1 & - & - \\
\hline $\mathrm{FOM}^{1}$ & $\mathrm{dBc} / \mathrm{Hz}$ & 189 & 189 & 174 & - & 189 & 190 & 193 \\
\hline
\end{tabular}

to the short-channel and narrow-channel effects [17]. Typically, transistors with a large channel width and a minimum gate length exhibit a reduced $V_{t}$, which is preferable for low-voltage operations. In this VCO topology, the fundamental limitation on the supply voltage is imposed by the threshold voltage of the cross-coupled transistors. To further reduce the supply voltage, the FBB technique is adopted as shown in Fig. 9. For a MOSFET device, the threshold voltage is governed by the body effect as [18]

$V_{t}=V_{t 0}+\left(\sqrt{2 q N_{A} \varepsilon_{s}} / C_{\mathrm{ox}}\right) \cdot\left(\sqrt{\left|2 \phi_{F}+V_{S B}\right|}-\sqrt{\left|2 \phi_{F}\right|}\right)$

where $V_{t 0}$ is the threshold voltage for $V_{S B}=0 \mathrm{~V}, \phi_{F}$ is a physical parameter with a typical value of $0.3 \mathrm{~V}, N_{A}$ is the substrate doping, and $\varepsilon_{s}$ is the permittivity of silicon. By applying a forward bias voltage to the body through a current-limiting resistor $R_{B}$, the effective threshold voltage is thus reduced while maintaining a minimum forward junction current between the body and the source terminals. The simulated effective threshold voltage and the drain current of a MOSFET with $W=35 \mu \mathrm{m}$ and $L=0.18 \mu \mathrm{m}$ are demonstrated in Fig. 10, indicating a threshold voltage reduction more than $100 \mathrm{mV}$ due to the FBB technique.

\section{CiRCUIT DESIGN}

Fig. 11 shows the design procedure of the high-performance VCO. The circuit design starts with the inductors $L_{1}$ and $L_{2}$. For simplification, both inductors are chosen to be identical as $L_{p}$, which are optimized for a high- $Q$ factor at the frequencies of interest. Once the inductance is determined, the required value of $\left(1 / C_{1}+2 / C_{2}\right)$ can be obtained from the designated oscillation frequency $\omega_{0}$ by (10)

$$
1 / C_{1}+2 / C_{2}=\omega_{0}^{2} L_{p}
$$

By defining the capacitance ratio $k=C_{1} / C_{2}$, the values of $C_{1}$ and $C_{2}$ are given by

$$
\begin{aligned}
& C_{1}=(1+2 k) /\left(\omega_{0}^{2} L_{p}\right) \\
& C_{2}=(1+2 k) /\left(k \omega_{0}^{2} L_{p}\right) .
\end{aligned}
$$

The circuit performance of the VCO is strongly influenced by the design parameter $k$. As indicated in (11) and (15), the required transconductance of the cross-coupled transistors and the associated output swing increase with $k$. Considering the design specifications such as the phase noise, output swing, tuning range, and power consumption, the optimum $k$ is thus determined and the circuit parameters including the capacitance values and the transistor aspect ratio can be calculated accordingly. Finally, a global optimization is performed and design iterations may be needed to satisfy the required circuit performance. Following the design procedures, the parameters of the VCO design are tabulated in Table I. Based on the narrowband approximation in (1) and (2), the calculated value of $R_{p}$ is $633 \Omega$ in this particular case. The resulting values for $g_{m} L_{p}$ and $R_{p}\left(C_{1}+C_{2}\right)$ are $1.2 \times 10^{-11}$ and $1.06 \times 10^{-9}$, respectively, which validate the assumption $g_{m} L_{p} \ll R_{p}\left(C_{1}+C_{2}\right)$ in the theoretical derivation of the proposed VCO.

\section{EXPERIMENTAL RESULTS}

The proposed VCO is implemented in a standard $1 \mathrm{P} 6 \mathrm{M}$ $0.18-\mu \mathrm{m}$ CMOS process provided by a commercial foundry. With an optimum layout for the RF performance, the $n$-channel MOSFETs in the deep n-well exhibit a maximum oscillation frequency $f_{\text {max }}$ up to $60 \mathrm{GHz}$. As for the passive components, top $\mathrm{AlCu}$ metallization layer of $2-\mu \mathrm{m}$ thickness is available for on-chip inductance while metal-insulator-metal (MIM) capacitors with oxide intermetal dielectric are also provided.

Fig. 12 shows a microphotograph of the fabricated circuit with a chip area of $0.55 \times 0.9 \mathrm{~mm}^{2}$ including the pads. In order to enhance the quality factor and to ensure the layout symmetry for fully differential operations, center-tapped spiral inductors 
with a metal width of $15 \mu \mathrm{m}$ are employed for the required inductance $L_{1}$ and $L_{2}$. In addition, the accumulation-type devices are used as the varactors. For test purposes, the VCO outputs are buffered by open-drain nMOS transistors to drive the external $50-\Omega$ load. To evaluate the circuit performance, the chip was mounted on an FR4 test board and measured with an Agilent E4407B spectrum analyzer.

When operating at a supply voltage of $0.6 \mathrm{~V}$, the VCO core consumes a dc power of $3 \mathrm{~mW}$. Fig. 13 shows the measured tuning characteristics. As the controlled voltage sweeps from 0 to $0.6 \mathrm{~V}$, the 5.6-GHz $\mathrm{VCO}$ exhibits a frequency tuning range of $8.1 \%$. The tuning range can be increased to $12.2 \%$ with a maximum controlled voltage of $1.8 \mathrm{~V}$. Fig. 14 shows the close-in output spectrum of the VCO with a controlled voltage of $0.3 \mathrm{~V}$. The measured output power and phase noise at $1-\mathrm{MHz}$ offset are $-1 \mathrm{dBm}$ and $-118.3 \mathrm{dBc} / \mathrm{Hz}$, respectively. For ultra-lowvoltage operations, the sensitivity to the supply voltage is also investigated. As $V_{D D}$ sweeps from 0.5 to $0.7 \mathrm{~V}$, the output characteristics of the VCO are illustrated in Fig. 15, exhibiting a reasonable performance deviation with respect to the variation in the supply voltage.

By employing the FBB technique, the VCO can operate at a supply voltage as low as $0.4 \mathrm{~V}$. With a supply voltage and a body bias of $0.4 \mathrm{~V}$, the $\mathrm{VCO}$ core consumes a dc power of $1 \mathrm{~mW}$. Fig. 16 shows the measured tuning characteristics, indicating a $6.4 \%$ tuning range as the controlled voltage sweeps from 0 to $0.4 \mathrm{~V}$. The close-in output spectrum of the VCO with a $0.2-\mathrm{V}$ controlled voltage is shown in Fig. 17. The measured phase noise is $-114.4 \mathrm{dBc} / \mathrm{Hz}$ at $1-\mathrm{MHz}$ offset, while the output power is $-9 \mathrm{dBm}$. The performance of the VCO is summarized in Table II along with the results from previously published low-voltage and low-power CMOS VCO circuits.

\section{CONCLUSION}

Using a $0.18-\mu \mathrm{m}$ CMOS technology, a VCO operating at a reduced supply voltage is presented. The performance of the fabricated circuit is characterized with a supply voltage from 0.6 to $0.4 \mathrm{~V}$. Due to the use of the capacitive feedback and the FBB technique, significant performance improvement in terms of output swing, frequency tuning range, and phase noise is demonstrated, making it extremely attractive for ultra-low-power and ultra-low-voltage RF applications.

\section{ACKNOWLEDGMENT}

The authors would like to thank the National Chip Implementation Center, Hsinchu, Taiwan, R.O.C., for chip fabrication, and K.-S. Chung, Realtek Semiconductor Corporation, Hsinchu, Taiwan, R.O.C., and Y.-C. Huang, National Taiwan University, Taipei, Taiwan, R.O.C., for technical support.

\section{REFERENCES}

[1] P. Choi et al., "An experimental coin-sized radio for extremely lowpower WPAN (IEEE 802.15.4) application at 2.4 GHz," IEEE J. SolidState Circuits, vol. 38, no. 12, pp. 2258-2268, Dec. 2003.

[2] S. Verma et al. "A 17-mW 0.66- $\mathrm{mm}^{2}$ direct-conversion receiver for $1-\mathrm{Mb} / \mathrm{s}$ cable replacement," IEEE J. Solid-State Circuits, vol. 40, no. 12, pp. 2547-2554, Dec. 2005.

[3] N.-J. Oh and S.-G. Lee, "A CMOS 868/915 MHz direct conversion ZigBee single-chip radio," IEEE Commun. Mag., vol. 43, no. 12, pp. 100-109, Dec. 2005.
[4] T.-K. Nguyen et al., "A low-power CMOS direct conversion receiver with 3-dB NF and 30-kHz flicker-noise corner for 915-MHz band IEEE 802.15.4 ZigBee standard," IEEE Trans. Microw. Theory Tech., vol. 54, no. 2, pp. 735-741, Feb. 2006.

[5] J. B. Kuo and J.-H. Lou, Low-Voltage CMOS VLSI Circuits. New York: Wiley, 1999.

[6] "International Technology Roadmap for Semiconductors," Semiconduct. Ind. Assoc., (2004 ed.). [Online]. Available: http://www.public. itrs.net/

[7] H.-H. Hsieh, K.-S. Chung, and L.-H. Lu, "Ultra-low-voltage mixer and VCO in 0.18- $\mu \mathrm{m}$ CMOS," in IEEE Radio Freq. Integr. Circuits Symp., Jun. 2005, pp. 167-170.

[8] K. Kwok and H. C. Luong, "Ultra-low-voltage high-performance CMOS VCOs using transformer feedback," IEEE J. Solid-State Circuits, vol. 40, no. 3, pp. 652-660, Mar. 2005.

[9] A. Fakhr, M. J. Deen, and H. deBruin, "Low-voltage, low-power and low phase noise $2.4 \mathrm{GHz}$ VCO for medical wireless telemetry," in Can. Elect. Comput. Eng. Conf., May 2004, vol. 3, pp. 1321-1324.

[10] A. H. Mostafa, M. N. El-Gamal, and R. A. Rafla, "A sub-1-V 4-GHz CMOS VCO and a $12.5-\mathrm{GHz}$ oscillator for low-voltage and high-frequency applications," IEEE Trans. Circuits Syst. II, Analog Digit. Signal Process., vol. 48, no. 10, pp. 919-926, Oct. 2001.

[11] M. Harada et al., "2-GHz RF front-end circuits in CMOS/SIMOX operating at an extremely low voltage of $0.5 \mathrm{~V}$," IEEE J. Solid-State Circuits, vol. 35, no. 12, pp. 2000-2004, Dec. 2000.

[12] N. Troedsson and H. Sjolamd, "An ultra low voltage $2.4 \mathrm{GHz}$ CMOS VCO," in IEEE Radio Wireless Conf., Aug. 2002, pp. 205-208.

[13] H. Wang, "Oscillator circuit having maximized signal power and reduced phase noise," U.S. Patent 6229 406, May 8, 2001.

[14] T. H. Lee, The Design of CMOS Radio-Frequency Integrated Circuits. New York: Cambridge Univ. Press, 1998.

[15] F. Gatta et al., "A 2-dB noise figure 900-MHz differential CMOS LNA,” IEEE J. Solid-State Circuits, vol. 36, no. 10, pp. 1444-1452, Oct. 2001.

[16] R. Aparicio and A. Hajimiri, "A noise-shifting differential Colpitts VCO,” IEEE J. Solid-State Circuits, vol. 37, no. 12, pp. 1728-1736, Dec. 2002.

[17] Y. Tsividis, Operation and Modeling of the MOS Transistor, 2nd ed. New York: Oxford Univ. Press, 1999.

[18] B. Razavi, Design of Analog CMOS Integrated Circuits. New York: McGraw-Hill, 2001.

[19] M. N. El-Gamal et al., "Very low-voltage (0.8 V) CMOS receiver frontend for $5 \mathrm{GHz}$ RF applications," Proc. Inst. Elect. Eng.-Circuits, Devices, Syst, vol. 149, no. 5/6, pp. 355-362, Oct.-Dec. 2002.

[20] M.-D. Tsai, Y.-H. Cho, and H. Wang, "A 5-GHz low phase noise differential Colpitts CMOS VCO," IEEE Microw. Wireless Compon. Lett., vol. 15 , no. 5, pp. 327-329, May 2005.

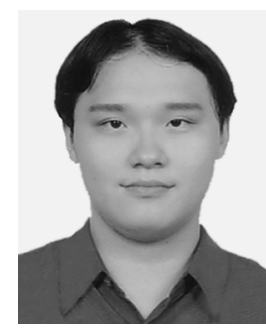

Hsieh-Hung Hsieh (S'05) was born in Taipei, Taiwan, R.O.C., in 1981. He received the B.S. degree in electrical engineering from National Taiwan University, Taipei, Taiwan, R.O.C., in 2004, and is currently working toward the Ph.D. degree in electronic engineering at National Taiwan University.

His research interests include the development of low-voltage and low-power RFICs, multiband wireless systems, RF testing, and monolithic microwave integrated circuit (MMIC) designs.

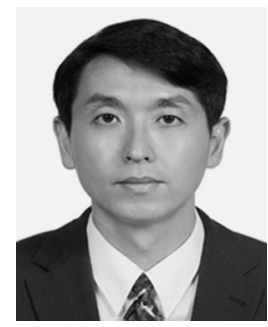

Liang-Hung Lu (M'02) was born in Taipei, Taiwan, R.O.C., in 1968. He received the B.S. and M.S. degrees in electronics engineering from National Chiao-Tung University, Hsinchu, Taiwan, R.O.C., in 1991 and 1993, respectively, and the Ph.D. degree in electrical engineering from The University of Michigan at Ann Arbor, in 2001.

During his graduate study, he was involved in SiGe HBT technology and monolithic microwave integrated circuit (MMIC) designs. From 2001 to 2002, he was with IBM, where he was involved with low-power and RFICs for silicon-on-insulator (SOI) technology. In August 2002, he joined the faculty of the Graduate Institute of Electronics Engineering and the Department of Electrical Engineering, National Taiwan University, Taipei, Taiwan, R.O.C., where he is currently an Associate Professor. His research interests include $\mathrm{CMOS} / \mathrm{BiCMOS} \mathrm{RF}$ and mixed-signal integrated-circuit designs. 